\title{
ON THE JACOBIAN OF MINIMAL GRAPHS IN $\mathbb{R}^{4}$
}

\author{
TH. HASANIS, A. SAVAS-HALILAJ, AND TH. VLACHOS
}

ABStRACT. We provide a characterization for complex analytic curves among two-dimensional minimal graphs in $\mathbb{R}^{4}$ via the Jacobian.

\section{INTRODUCTION}

The classical theorem of S. Bernstein 2] states that the only entire minimal graphs in the Euclidean space $\mathbb{R}^{3}$ are planes. Equivalently, if $u: \mathbb{R}^{2} \rightarrow \mathbb{R}$ is an entire smooth solution of the differential equation

$$
\operatorname{div}\left(\frac{\operatorname{grad} u}{\sqrt{1+|\operatorname{grad} u|^{2}}}\right)=0,
$$

then $u$ is an affine function. It was conjectured for a long time that the theorem of Bernstein holds in any dimension. However, for $n=3$, its validity was proved by E. De Giorgi [5], for $n=4$ by F. Almgren [1] and for $n=5,6,7$ by J. Simons [13]. It was a big surprise when E. Bombieri, E. De Giorgi and E. Giusti [3] proved that, for $n \geq 8$, there are entire solutions of the minimal surface equation other than the affine ones.

In this paper we study minimal surfaces $M^{2}$ which arise as graphs over vector valued maps $f: \mathbb{R}^{2} \rightarrow \mathbb{R}^{2}, f=\left(f_{1}, f_{2}\right)$, that is

$$
M^{2}=G_{f}:=\left\{\left(x, y, f_{1}(x, y), f_{2}(x, y)\right) \in \mathbb{R}^{4}:(x, y) \in \mathbb{R}^{2}\right\} .
$$

There are plenty of complete minimal graphs in $\mathbb{R}^{4}$, other than the planes. More precisely, if $f: \mathbb{C} \rightarrow \mathbb{C}$ is any entire holomorphic or anti-holomorphic function, then the graph $G_{f}$ of $f$ in $\mathbb{C}^{2}=\mathbb{R}^{4}$ is a minimal surface and is called a complex analytic curve. It should be noticed that R. Osserman in 9, has constructed examples of complete minimal two dimensional graphs in $\mathbb{R}^{4}$, which are not complex analytic curves with respect to any orthogonal complex structure on $\mathbb{R}^{4}$. For instance, the graph $G_{f}$ over the map $f: \mathbb{R}^{2} \rightarrow \mathbb{R}^{2}$, given by

$$
f(x, y)=\frac{1}{2}\left(e^{x}-3 e^{-x}\right)\left(\cos \frac{y}{2},-\sin \frac{y}{2}\right), \quad(x, y) \in \mathbb{R}^{2},
$$

is such an example. It is worth noticing that the Jacobian $J_{f}:=\operatorname{det}(d f)$ of $f$ given by $J_{f}=-\left(e^{2 x}-9 e^{-2 x}\right) / 8$ takes every real value.

The problem that we deal with in this article, is to find under which geometric conditions the minimal graph of an entire vector valued map $f: \mathbb{R}^{2} \rightarrow \mathbb{R}^{2}, f=$ $\left(f_{1}, f_{2}\right)$, is a complex analytic curve. The first result was obtained by S.S. Chern

2000 Mathematics Subject Classification. Primary 53C42.

Key words and phrases. Minimal surface, Bernstein type theorem, Jacobian.

This work was written during the second author's stay at the University of Ioannina as a research fellow of the "Foundation for Education and European Culture". 
and R. Osserman [4], where they proved that if the differential $d f$ of $f$ is bounded, then $G_{f}$ must be a plane. A few years later, L. Simon [12] obtained a much more general result by proving that if one of $f_{1}, f_{2}$ has bounded gradient, then $f$ is affine. Later on, R. Schoen [11 obtained a Bernstein type result by imposing the assumption that $f: \mathbb{R}^{2} \rightarrow \mathbb{R}^{2}$ is a diffeomorphism. Moreover, L. Ni $[8$ has derived a result of Bernstein type under the assumption that $f$ is an area-preserving map, that is the Jacobian $J_{f}$ satisfies $J_{f}=1$. This result was generalized by the authors in a previous paper [7], just by assuming that $J_{f}$ is bounded.

Another interesting class of minimal surfaces in $\mathbb{R}^{4}$ can be obtained by considering graphs over vector valued maps of the form $f=\operatorname{grad} u$, where $u: U \subset \mathbb{R}^{2} \rightarrow \mathbb{R}$ is a smooth function and $U$ an open subset of $\mathbb{R}^{2}$. It can be shown that the graph over $f=\operatorname{grad} u: U \rightarrow \mathbb{R}^{2}$ is a minimal surface if and only if the function $u$ satisfies the so called Special Lagrangian Equation

$$
\cos \theta \Delta u=\sin \theta(\operatorname{det} \operatorname{Hess} u-1),
$$

for some real constant $\theta$. L. Fu [6], proved that the entire solutions of Special Lagrangian Equation are only the harmonic functions or the quadratic polynomials, which means that the entire minimal graph of $\operatorname{grad} u$ is either a complex analytic curve or a plane.

In this paper, we prove the following result of Bernstein type, from which known results due to $\mathrm{R}$. Schoen [11], L. Fu [6], L. Ni [8], and the authors [7] follow as a consequence.

Theorem 1.1. Let $f: \mathbb{R}^{2} \rightarrow \mathbb{R}^{2}$ be an entire smooth vector valued map such that its graph $G_{f}$ is a minimal surface in $\mathbb{R}^{4}$. Assume that $G_{f}$ is not a plane. Then, the graph $G_{f}$ over $f$ is a complex analytic curve if and only if the Jacobian $J_{f}$ of $f$ does not take every real value. In particular, if $G_{f}$ is a complex analytic curve, then the Jacobian $J_{f}$ takes every real value in $(0,+\infty)$ or in $[0,+\infty)($ resp. $(-\infty, 0)$ or in $(-\infty, 0])$, if $f$ is holomorphic (resp. anti-holomorphic).

\section{Preliminaries}

At first we set up the notation and recall some basic facts about minimal surfaces in $\mathbb{R}^{4}$. Let $U$ be an open subset of $\mathbb{R}^{2}$ and $f: U \rightarrow \mathbb{R}^{2}, f=\left(f_{1}, f_{2}\right)$, a smooth vector valued map. Then, its graph can be represented by the map $X: U \rightarrow \mathbb{R}^{4}$, given by

$$
X(x, y)=\left(x, y, f_{1}(x, y), f_{2}(x, y)\right), \quad(x, y) \in U .
$$

Denote by $g_{11}, g_{12}=g_{21}$ and $g_{22}$ the coefficients of the first fundamental form, which are given by

$$
g_{11}=1+\left|f_{x}\right|^{2}, \quad g_{12}=g_{21}=\left\langle f_{x}, f_{y}\right\rangle, \quad g_{22}=1+\left|f_{y}\right|^{2},
$$

where $\langle$,$\rangle stands for the Euclidean inner product.$

Suppose, now, that $\xi$ is a unit vector field normal to the surface $G_{f}$. Let $b_{11}(\xi)$, $b_{12}(\xi)=b_{21}(\xi)$ and $b_{22}(\xi)$ be the coefficients of the second fundamental form with respect to the direction $\xi$, that is

$$
b_{11}(\xi)=\left\langle X_{x x}, \xi\right\rangle, \quad b_{12}(\xi)=b_{21}(\xi)=\left\langle X_{x y}, \xi\right\rangle, \quad b_{22}(\xi)=\left\langle X_{y y}, \xi\right\rangle .
$$

The mean curvature $H(\xi)$, with respect to the normal direction $\xi$, is defined by the formula

$$
H(\xi):=\frac{g_{22} b_{11}(\xi)-2 g_{12} b_{12}(\xi)+g_{11} b_{22}(\xi)}{2\left(g_{11} g_{22}-g_{12}^{2}\right)} .
$$


The surface $G_{f}$ is called minimal if it is a critical point of the area functional. It can be proved that $G_{f}$ is minimal if and only if the mean curvature, with respect to any unit normal vector field along $G_{f}$, is zero. A simple computation shows that minimality of $G_{f}$ is expressed by the differential equation

$$
\left(1+\left|f_{y}\right|^{2}\right) f_{x x}-2\left\langle f_{x}, f_{y}\right\rangle f_{x y}+\left(1+\left|f_{x}\right|^{2}\right) f_{y y}=0
$$

the so called minimal surface equation.

It is a fact of central importance that any surface can be parametrized (at least locally) by isothermal parameters. This means that there exists a local diffeomorphism $F(u, v)=(x(u, v), y(u, v))$ such that the coefficients of the first fundamental form of the new parametrization

$$
X(u, v)=\left(x(u, v), y(u, v), f_{1}(x(u, v), y(u, v)), f_{2}(x(u, v), y(u, v))\right)
$$

become

$$
g_{11}(u, v)=g_{22}(u, v)=E(u, v) \text { and } g_{12}(u, v)=g_{21}(u, v)=0 .
$$

Set

$$
\varphi(u, v):=f_{1}(x(u, v), y(u, v)) \text { and } \psi(u, v):=f_{2}(x(u, v), y(u, v)) .
$$

Then, in isothermal parameters, the minimal surface equation is equivalent to

$$
\begin{aligned}
x_{u u}+x_{v v} & =0=y_{u u}+y_{v v}, \\
\varphi_{u u}+\varphi_{v v} & =0=\psi_{u u}+\psi_{v v} .
\end{aligned}
$$

Furthermore, the complex valued functions $\phi_{k}: U \subseteq \mathbb{C} \rightarrow \mathbb{C}, k=1,2,3,4$, given by

$$
\begin{cases}\phi_{1}=x_{u}-i x_{v}, & \phi_{2}=y_{u}-i y_{v} \\ \phi_{3}=\varphi_{u}-i \varphi_{v}, & \phi_{4}=\psi_{u}-i \psi_{v}\end{cases}
$$

are holomorphic and satisfy

$$
\phi_{1}^{2}+\phi_{2}^{2}+\phi_{3}^{2}+\phi_{4}^{2}=0 .
$$

Assume, now, that $f: \mathbb{R}^{2} \rightarrow \mathbb{R}^{2}$ is an entire solution of the minimal surface equation. In this case, R. Osserman [9, Theorem 5.1] proved the following result which will be the main tool for the proof of our results.

Theorem 2.1. Let $f: \mathbb{R}^{2} \rightarrow \mathbb{R}^{2}$ be an entire solution of the minimal surface equation. Then there exist real constants $a, b$, with $b>0$, and a non-singular transformation

$$
x=u, \quad y=a u+b v,
$$

such that $(u, v)$ are global isothermal parameters for the surface $G_{f}$.

\section{Proof of the theorem}

Let $f=\left(f_{1}, f_{2}\right)$ be an entire solution of the minimal surface equation

$$
\left(1+\left|f_{y}\right|^{2}\right) f_{x x}-2\left\langle f_{x}, f_{y}\right\rangle f_{x y}+\left(1+\left|f_{x}\right|^{2}\right) f_{y y}=0 .
$$

Then its graph,

$$
G_{f}=\left\{\left(x, y, f_{1}(x, y), f_{2}(x, y)\right) \in \mathbb{R}^{4}:(x, y) \in \mathbb{R}^{2}\right\},
$$

is a minimal surface. According to Theorem 2.1, we can introduce global isothermal parameters, via a non-singular transformation

$$
x=u, \quad y=a u+b v,
$$


where $a, b$ are real constants with $b>0$. Now, the minimal surface $G_{f}$ is parametrized by the map

$$
X(u, v)=(u, a u+b v, \varphi(u, v), \psi(u, v)),
$$

where

$$
\varphi(u, v):=f_{1}(u, a u+b v) \text { and } \psi(u, v):=f_{2}(u, a u+b v) .
$$

Set $\Phi=(\varphi, \psi)$. Because of the relation

$$
\frac{\partial(\varphi, \psi)}{\partial(u, v)}=\frac{\partial\left(f_{1}, f_{2}\right)}{\partial(x, y)} \frac{\partial(x, y)}{\partial(u, v)}
$$

for the Jacobians, we obtain

$$
J_{\Phi}=b J_{f}
$$

Since $(u, v)$ are isothermal parameters and taking into account that $G_{f}$ is minimal, it follows that the functions $\varphi$ and $\psi$ are harmonic, that is

$$
\varphi_{u u}+\varphi_{v v}=0=\psi_{u u}+\psi_{v v} .
$$

Then, the complex valued functions $\phi_{k}: \mathbb{C} \rightarrow \mathbb{C}, k=1,2,3,4$, given by

$$
\begin{cases}\phi_{1}=1, & \phi_{2}=a-i b \\ \phi_{3}=\varphi_{u}-i \varphi_{v}, & \phi_{4}=\psi_{u}-i \psi_{v}\end{cases}
$$

are holomorphic and satisfy

$$
\phi_{1}^{2}+\phi_{2}^{2}+\phi_{3}^{2}+\phi_{4}^{2}=0 .
$$

Proof of Theorem 1.1. Assume that the graph $G_{f}$ of $f(x, y)=\left(f_{1}(x, y), f_{2}(x, y)\right)$, $(x, y) \in \mathbb{R}^{2}$, is a minimal surface which is not a plane.

Suppose now that $J_{f}$ does not take every real value. We shall prove that $G_{f}$ is a complex analytic curve. Arguing indirectly, we assume that $G_{f}$ is not a complex analytic curve. Then, equation (3.2) can be written equivalently in the form

$$
\left(\phi_{3}-i \phi_{4}\right)\left(\phi_{3}+i \phi_{4}\right)=-d
$$

where

$$
d=1+(a-i b)^{2} .
$$

We claim that $d \neq 0$. Assume to the contrary that $d=0$. Then, $a=0, b=1$ and consequently $(x, y)$ are isothermal parameters. Furthermore, from (3.3) we obtain $\varphi_{3}= \pm i \varphi_{4}$, or equivalently,

$$
\frac{\partial f_{1}}{\partial x}-i \frac{\partial f_{1}}{\partial y}= \pm i\left(\frac{\partial f_{2}}{\partial x}-i \frac{\partial f_{2}}{\partial y}\right) .
$$

From (3.4) we readily deduce that $f=f_{1}+i f_{2}$ is holomorphic or anti-holomorphic, which is a contradiction. Hence $d \neq 0$.

By virtue of (3.3), we see that $\varphi_{3}-i \varphi_{4}, \varphi_{3}+i \varphi_{4}$ are entire nowhere vanishing holomorphic functions. Define the complex valued function $h: \mathbb{C} \rightarrow \mathbb{C}$, by

$$
h=\phi_{3}-i \phi_{4} .
$$

We point out that $h$ is holomorphic, non-constant and nowhere vanishing. Combining (3.3) with (3.5), we get

$$
\phi_{3}=\frac{1}{2}\left(h-\frac{d}{h}\right) \text { and } \phi_{4}=\frac{i}{2}\left(h+\frac{d}{h}\right) .
$$

Bearing in mind (3.1), it follows that the imaginary part of $\phi_{3} \bar{\phi}_{4}$ is given by

$$
\operatorname{Im}\left(\phi_{3} \bar{\phi}_{4}\right)=\varphi_{u} \psi_{v}-\varphi_{v} \psi_{u}=J_{\Phi} .
$$


On the other hand, from (3.6) we get

$$
\operatorname{Im}\left(\phi_{3} \bar{\phi}_{4}\right)=\frac{1}{4}\left(-|h|^{2}+\frac{|d|^{2}}{|h|^{2}}\right) .
$$

Thus, taking into account the relation $J_{\Phi}=b J_{f}$, we have

$$
J_{f}=\frac{1}{4 b}\left(-|h|^{2}+\frac{|d|^{2}}{|h|^{2}}\right) .
$$

Since $h$ is an entire and non-constant holomorphic function, by Picard's Theorem, there are sequences $\left\{z_{n}\right\}_{n \in \mathbb{N}}$ and $\left\{w_{n}\right\}_{n \in \mathbb{N}}$ of complex numbers such that $\left|h\left(z_{n}\right)\right| \rightarrow$ $\infty$ and $\left|h\left(w_{n}\right)\right| \rightarrow 0$. Consequently, $J_{f}\left(z_{n}\right) \rightarrow-\infty$ and $J_{f}\left(w_{n}\right) \rightarrow \infty$. Thus, $J_{\Phi}\left(\mathbb{R}^{2}\right)=\mathbb{R}$, which contradicts to our assumptions. Therefore, $G_{f}$ must be a complex analytic curve.

Conversely, assume that $G_{f}$ is a complex analytic curve which is not a plane. Then, the complex valued function $f=f_{1}+i f_{2}$ is holomorphic or anti-holomorphic. We introduce the complex variable $z=x+i y$. An easy computation shows that

$$
J_{f}=\left|f_{z}\right|^{2}-\left|f_{\bar{z}}\right|^{2} .
$$

Hence, $J_{f} \geq 0$ if $f$ is holomorphic and $J_{f} \leq 0$ if $f$ is anti-holomorphic. In either case, $J_{f}$ does not take every real value.

Asssume at first that $f$ is holomorphic. Then,

$$
J_{f}=\left|f_{z}\right|^{2} .
$$

By minimality, we obtain that $f_{z}$ is an entire holomorphic function. Moreover $f_{z}$ cannot be constant, since otherwise $f$ is affine and $G_{f}$ a plane. Consequently, appealing to Picard's Theorem, the range of $f_{z}$ is the whole complex plane $\mathbb{C}$, or the plane minus a single point. Thus, the range of $J_{f}$ must be $(0,+\infty)$ or $[0,+\infty)$.

Suppose, now, that $f$ is anti-holomorphic. Then,

$$
J_{f}=-\left|f_{\bar{z}}\right|^{2}
$$

and $f_{\bar{z}}$ is an entire anti-holomorphic function. Arguing as above, we deduce that range of $J_{f}$ must be $(-\infty, 0)$ or $(-\infty, 0]$.

\section{Applications}

In this last section, we reobtain some known Bernstein type theorems for entire vector valued maps $f: \mathbb{R}^{2} \rightarrow \mathbb{R}^{2}$ using the method developed here. The following result was first obtained by L. Simon [12].

Corollary 4.1. Let $f: \mathbb{R}^{2} \rightarrow \mathbb{R}^{2}, f=\left(f_{1}, f_{2}\right)$ be an entire solution of the minimal surface equation, such that one of $f_{1}, f_{2}$ has bounded gradient. Then, $f$ is an affine map.

Proof. Without loss of generality, let us assume that $f_{1}$ has bounded gradient. By virtue of Theorem 2.1, we can introduce global isothermal parameters $(u, v)$, via the non-singular transformation

$$
x=u, \quad y=a u+b v,
$$

where $a, b$ are real constants with $b>0$. Then, the graph $G_{f}$ is parametrized via the map

$$
X(u, v)=\left(u, a u+b v, f_{1}(u, a u+b v), f_{2}(u, a u+b v)\right) .
$$


Consider the real valued functions $\varphi, \psi: \mathbb{R}^{2} \rightarrow \mathbb{R}$, given by

$$
\varphi(u, v):=f_{1}(u, a u+b v), \quad \psi(u, v):=f_{2}(u, a u+b v) .
$$

Since $(u, v)$ are isothermal parameters, $\varphi, \psi$ are harmonic functions. Moreover, the complex valued functions $\phi_{k}: \mathbb{C} \rightarrow \mathbb{C}, k=1,2,3,4$, given by

$$
\begin{cases}\phi_{1}=1, & \phi_{2}=a-i b \\ \phi_{3}=\varphi_{u}-i \varphi_{v}, & \phi_{4}=\varphi_{u}-i \psi_{v},\end{cases}
$$

are holomorphic and satisfy

$$
\phi_{1}^{2}+\phi_{2}^{2}+\phi_{3}^{2}+\phi_{4}^{2}=0 .
$$

A simple calculation shows that

$$
\frac{\partial \varphi}{\partial u}=\frac{\partial f_{1}}{\partial x}+a \frac{\partial f_{1}}{\partial y}, \quad \frac{\partial \varphi}{\partial v}=b \frac{\partial f_{1}}{\partial y} .
$$

Since $f_{1}$ has bounded gradient, it follows that $\varphi$ has also bounded gradient. Because $\varphi_{u}$ and $\varphi_{v}$ are harmonic functions, by Liouville's Theorem it follows that they must be constants. Hence $\phi_{3}$ is a constant complex valued function. By virtue of (4.1), we obtain that $\phi_{4}$ must also be constant. Hence $G_{f}$ is a plane and $f$ is an affine function.

The following result due to R. Schoen [11] is a consequence of Theorem 1.1.

Corollary 4.2. Let $f: \mathbb{R}^{2} \rightarrow \mathbb{R}^{2}$ be an entire solution of the minimal surface equation. If $f$ is a diffeomorphism, then $f$ is an affine map.

Proof. Since $f$ is a diffeomorphism, it follows that $J_{f}>0$ if $f$ is orientation preserving, or $J_{f}<0$ if $f$ is orientation reversing. Then, by Theorem 1.1, $f$ must be holomorphic or anti-holomorphic. Thus, $f$ is an entire conformal diffeomorphism. By a classical theorem of Complex Analysis [10, p. 388], $f$ must be an affine map.

Finally, we provide an alternative proof of the result due to L. Fu [6], based on Theorem 1.1.

Corollary 4.3. Let $u: \mathbb{R}^{2} \rightarrow \mathbb{R}$ be an entire solution of the Special Lagrangian Equation

$$
\cos \theta \Delta u=\sin \theta(\operatorname{det} \operatorname{Hess} u-1),
$$

where $\theta$ is a real constant. Then, either $u$ is a harmonic function or $u$ is a quadratic polynomial.

Proof. Consider the entire vector valued map $f=\operatorname{grad} u: \mathbb{R}^{2} \rightarrow \mathbb{R}^{2}$. Since $u$ satisfies the Special Lagrangian Equation, the graph of $f$ is a minimal surface in $\mathbb{R}^{4}$. Note that in this case

$$
J_{f}=\operatorname{det} \operatorname{Hess} u=u_{x x} u_{y y}-u_{x y}^{2} .
$$

Suppose at first that there is a point $\left(x_{0}, y_{0}\right) \in \mathbb{R}^{2}$ such that $J_{f}\left(x_{0}, y_{0}\right)=1$. Then, at this point the Laplacian of $u$ satisfies

$$
\Delta u\left(x_{0}, y_{0}\right)=u_{x x}\left(x_{0}, y_{0}\right)+u_{y y}\left(x_{0}, y_{0}\right) \neq 0 .
$$

Consequently, $\theta=\frac{\pi}{2}$ and $J_{f} \equiv 1$. By Theorem 1.1, $f$ must be affine and thus $u$ a quadratic polynomial. 
Assume now that $J_{f} \neq 1$. Then, either $J_{f}>1$ or $J_{f}<1$. If $J_{f}>1$, then, according to Theorem 1.1, $f$ must be an affine map and so $u$ is a quadratic polynomial. If $J_{f}<1$, then, by virtue of Theorem 1.1 , we deduce that $J_{f} \leq 0$ and $f$ is an anti-holomorphic function. Appealing to Cauchy-Riemann equations, it follows that $u$ is a harmonic function.

\section{REFERENCES}

[1] F. Almgren, Some interior regularity theorems for minimal surfaces and an extension of Bernstein's theorem, Ann. of Math., 84 (1966), 277-292.

[2] S. Bernstein, Sur un théorème de géométrie et ses applications aux équations aux dérivées partielles du type elliptique, Comm. de la Soc. Math. de Kharkov 15 (1915 - 1917), 38-45.

[3] E. Bombieri, E De Giorgi and E. Giusti, Minimal cones and the Bernstein conjecture, Invent. Math. 7 (1969), 243-268.

[4] S.S. Chern and R. Osserman, Complete minimal surfaces in Euclidean n-space, J. d'Analyse Math. 19 (1967), 15-34.

[5] E. De Giorgi, Una extensione del teorema di Bernstein, Ann. Scuola Norm. Sup. Pisa 19 (1965), 79-85.

[6] L. Fu, An analogue of Bernstein's theorem, Houston J. Math. 24 (1998), 415-419.

[7] Th. Hasanis, A. Savas-Halilaj and Th. Vlachos, Minimal graphs in $\mathbb{R}^{4}$ with bounded Jacobians, Proc. Amer. Math. Soc. 137 (2009), 3463-3471.

[8] L. Ni, A Bernstein type theorem for minimal volume preserving maps, Proc. Amer. Math. Soc. 130 (2002), 1207-1210.

[9] R. Osserman, A Survey of Minimal Surfaces, Van Nostrand-Reinhold, New York, 1969.

[10] B. Palka, An Introduction to Complex Function Theory, Springer-Verlag, New York, 1995.

[11] R. Schoen, The role of harmonic mappings in rigidity and deformation problems, Complex Geometry (Osaka 1990), 179-200, Lecture Notes in Pure and Appl. Math. 143, Dekker, New York, 1993.

[12] L. Simon, A Hölder estimate for quasiconformal maps between surfaces in euclidean space, Acta Math. 139 (1977), 19-51.

[13] J. Simons, Minimal varieties in Riemannian manifolds, Ann. of Math. (2) 88 (1968), 62-105.

Department of Mathematics, University of Ioannina, 45110, Ioannina, Greece

E-mail address: thasanis@uoi.gr

Department of Mathematics and Statistics, University of Cyprus, P.O. Box 20537, 1678, Nicosia, Cyprus

E-mail address: halilaj.andreas@ucy.ac.cy

Department of Mathematics, University of Ioannina, 45110, Ioannina, Greece

E-mail address: tvlachos@uoi.gr 